\title{
Nhân tố ảnh hưởng sự sáng tạo của nhân viên tại trường đại học công lập Thành phố Hồ Chí Minh
}

\section{Factors affecting the creativity of employees at public universities in Ho Chi Minh City}

\author{
Nguyễn Thu Ngân ${ }^{1}$, Trần Thị Thanh Phương ${ }^{2 *}$ \\ ${ }^{1}$ Đại học Quốc gia Thành phố Hồ Chí Minh, Việt Nam \\ ${ }^{2}$ Trường Đại học Kinh tế Thành phố Hồ Chí Minh, Việt Nam \\ *Tác giả liên hệ, Email: phuong.tran@ueh.edu.vn
}

\section{THÔNG TIN}

DOI: $10.46223 /$ HCMCOUJS. econ.vi.16.3.1305.2021

Ngày nhận: 04/11/2020

Ngày nhận lại: 10/01/2021

Duyệt đăng: 11/01/2021

\section{Tù khóa:}

động lực làm việc; mô hình SEM; nhân viên; sự sáng tạo

Keywords:

motivation to work; SEM; employees; creativity;

\section{TÓM TÁT}

Nghiên cứu này nhằm xác định các nhân tố ảnh hưởng đến sự sáng tạo của nhân viên làm việc tại các trường đại học công lập ở Thành phố Hồ Chí Minh. Dữ liệu được thu thập từ 247 nhân viên làm việc từ 10 trường Đại học công lập. Nghiên cứu phân tích dựa trên mô hình cấu trúc tuyến tính hiệp phương sai (CB-SEM). Kết quả cho thấy bốn nhân tố: i) Sự hỗ trợ của tổ chức, ii) Tính chủ động cá nhân, iii) Động lực nội tại, iv) Ý nghĩa công việc tác động mạnh mẽ đến sự sáng tạo của nhân viên làm việc tạc các trường đại học công lập tại Thành phố Hồ Chí Minh. Do đó, những phát hiện của bài nghiên cứu này sẽ giúp các nhà quản lý tại các trường đại học xây dựng giải pháp tăng cường sự sáng tạo của nhân viên.

\section{ABSTRACT}

This study aims to identify factors that influence the creativity of employees working at public universities in Ho Chi Minh City. The data was collected from 247 employees working from 10 public universities. A covariance-based structural equation model (CB-SEM) was applied for analysis. The results show that four factors: i) The support of the organization, ii) Personal autonomy, iii) Internal motivation, iv) The meaning of work strongly influences the creativity of employees working at public universities in Ho Chi Minh City. The findings of this study help managers at universities build future solutions to enhance employee creativity.

\section{Giới thiệu}

Nguồn nhân lực là động lực của mọi sự sáng tạo, đổi mới trong tổ chức và có ý nghĩa chiến lược tạo ra lợi ích cho doanh nghiệp (Luecke \& Katz, 2004; N. D. Nguyen \& Le, 2017). Hiện nay không có nhiều nghiên cứu về sự sáng tạo của nhân viên tại các đơn vị sự nghiệp công lập với cơ chế hoạt động hoàn toàn khác biệt so với một doanh nghiệp sản xuất, kinh doanh (Ha \& Nguyen, 2015). Đơn vị sự nghiệp công lập là nơi mà sự sáng tạo của nhân viên chưa được khai thác và ít có những đổi mới và chuyển đổi tích cực trong công việc. Lý do là đơn vị sự nghiệp công lập có cơ chế quản lý đặc thù và cơ chế thu nhập cố định. Điều này tạo 
nên một khuyết điểm rất lớn là chú trọng thu nhập của những người có cùng thâm niên, và trình độ học vấn, và không quan tâm đến năng lực làm việc cũng như kết quả công việc của nhân viên và do đó không đảm bảo nguyên tắc phân phối theo lao động $(\mathrm{N}$. D. Nguyen \& Le, 2017; Tran, 2013). Chính vì vậy, việc nâng cao sự sáng tạo của nhân viên tại các đơn vị sự nghiệp công lập đặc biệt là tại các trường đại học công lập đã trở thành một vấn đề cấp thiết để nâng cao sự hiệu quả làm việc.

Từ những nhận định trên, nghiên cứu này tìm hiểu về các yếu tố tác động đến năng lực sáng tạo của nhân viên làm việc tại các trường đại học công lập ở Thành phố Hồ Chí Minh, bao gồm các yếu tố thuộc về môi trường làm việc và các yếu tố thuộc về cá nhân. Mục tiêu nghiên cứu là đánh giá sự ảnh hưởng của các yếu tố động lực nội tại, sự hỗ trợ của tổ chức, tính chủ động cá nhân, ý nghĩa công việc đến sự sáng tạo của nhân viên làm việc tại các trường đại học công lập tại Thành phố Hồ Chí Minh.

Cấu trúc của bài nghiên cứu như sau: Phần 1 giới thiệu. Phần 2 trình bày về cơ sở lý thuyết về các nhân tố ảnh hưởng sự sáng tạo của nhân viên và đề xuất mô hình nghiên cứu. Phần 3 giới thiệu về phương pháp nghiên cứu. Phần 4 trình bày kết quả nghiên cứu và kiểm định giả thuyết. Phần 5 đề xuất kết luận và hàm ý quản trị.

\section{Tổng quan lý thuyết và các nghiên cứu trước đây liên quan đến các nhân tố ảnh hưởng sự sáng tạo của nhân viên}

Sự sáng tạo là việc tạo ra một sản phẩm hoặc giải pháp mới lạ, phù hợp đồng thời giải pháp phải là giải pháp mở, có nghĩa là không quá cụ thể, không có sự gò bó, cứng nhắc mà phải linh hoạt. Các giải pháp, phương án được đưa ra không hẳn là mới hoàn toàn, nhiều khi chỉ mới một phần nhưng phải phù hợp để hoàn thành nhiệm vụ, giải quyết được vấn đề. Như vậy, nó mới có giá trị, đúng đắn, khả thi và phù hợp với mục tiêu cụ thể đã đề ra của tổ chức (Amabile, 2012). Sau này xuất hiện một định nghĩa khá mới về sự sáng tạo được đưa ra bởi (Dawson \& Andriopoulos, 2014), sự sáng tạo của nhân viên là quá trình mà các ý tưởng mới và hữu dụng được hình thành.

Sự sáng tạo của nhân viên là việc tạo ra những sản phẩm, dịch vụ, thủ tục, và quá trình mới hữu ích và có giá trị (Amabile, 1983, 1988, 1997; Amabile, Conti, Coon, Lazenby, \& Herron, 1996). Tổng hợp của các nghiên cứu trước đây đã phân loại các yếu tố ảnh hưởng sự sáng tạo của nhân viên thành hai nhóm: các yếu tố thuộc về cá nhân và các yếu tố thuộc về tổ chức.

Các yếu tố thuộc về cá nhân như: động lực nội tại, tính cách, kiến thức, phong cách tư duy, phong cách làm việc, sự tự chủ, kỹ năng tư duy sáng tạo (Amabile, 2012; Amabile, Barsade, Mueller, \& Staw, 2005; Shalley, Zhou, \& Oldham, 2004; Woodman, Sawyer, \& Griffin, 1993). Các yếu tố thuộc về môi trường tổ chức như: sự hỗ trợ của tổ chức về sáng tạo, phong cách lãnh đạo, động lực bên ngoài, phần thưởng, văn hóa tổ chức, các nguồn lực, sự tự do, sự phức tạp của công việc (DiLiello \& Houghton, 2006; Eder \& Sawyer, 2008; Eisenberger, Huntington, Hutchison, \& Sowa, 1986; Houghton \& DiLiello, 2010; Shalley et al., 2004).

Amabile (1983) tìm ra ba nhóm nhân tố chính ảnh hưởng đến sự sáng tạo của nhân viên: 1) Kỹ năng chuyên môn, (domain- relevent skills): kiến thức, yêu cầu về kỹ thuật, tài năng. Những yếu tố này phụ thuộc vào: khả năng nhận thức bẩm sinh, kỹ năng vận động và tri giác bẩm sinh, giáo dục chính thống cũng như không chính thống; 2) Kỹ năng tư duy sáng tạo Những kỹ năng sáng tạo (creative- relevent skills): phong cách nhận thức thích hợp, kiến thức tiềm ẩn hoặc rõ ràng để giải thích những ý tưởng mới lạ, phong cách làm việc có lợi. Những yếu tố này phụ thuộc vào: đào tạo, kinh nghiệm hình thành ý tưởng, tính cách cá nhân; 3) Động lực làm việc. Trong đó, động lực làm việc bao gồm động lực nội tại (sở thích, sự liên quan, tò mò, thích 
thú, thử thách) và động lực bên ngoài (mong muốn đạt thành quả, khen thưởng). Hai loại động lực này có sự liên quan với nhau nhưng Amabile và cộng sự (1996) cho rằng động lực nội tại dẫn đến sáng tạo tác động nhiều hơn động lực bên ngoài.

Amabile và cộng sự (1996) bổ sung các yếu tố thuộc về môi trường tổ chức có ảnh hưởng đến sự sáng tạo mà những lý thuyết trước đây chưa đề cập đến đó là: động lực đổi mới của tổ chức, các nguồn lực và các hoạt động thực tiễn quản lý. Nghiên cứu này xây dựng khung công cụ đánh giá các yếu tố môi trường tổ chức ảnh hưởng đến sự sáng tạo của nhân viên (KEYS Environment Scale) gồm có 05 nhóm: khuyến khích sáng tạo (sự khuyến khích của tổ chức, sự khuyến khích của người quản lý, sự hỗ trợ của nhóm), sự tự chủ/tự do, nguồn lực đầy đủ, áp lực (thử thách công việc, áp lực khối lượng công việc), trở ngại của tổ chức. Hầu hết các yếu tố này đều tác động cùng chiều với sự sáng tạo, riêng hai yếu tố áp lực khối lượng công việc và trở ngại của tổ chức là tác động ngược chiều với sự sáng tạo. Tuy nhiên, các nghiên cứu của Amabile và cộng sự $(1996,2005)$ chủ yếu thực hiện tại các doanh nghiệp sản xuất kinh doanh, các ngành công nghiệp, sản xuất truyền thống, ngân hàng, hàng tiêu dùng; ngành công nghiệp hóa học, công nghệ cao và hàng tiêu dùng.

Tổng kết từ các công trình nghiên cứu, bốn yếu tố ảnh hưởng nhiều đến sự sáng tạo của nhân viên và được nghiên cứu nhiều trong những ngành nghề và lĩnh vực khác nhau: i) Sự hỗ trợ của tổ chức, ii) Tính chủ động cá nhân, iii) Động lực nội tại, iv) Ý nghĩa công việc.

\subsection{Sự hỗ trợ của tổ chức (Perceived organizational support, HT)}

Blau (1964) phát triển lý thuyết trao đổi xã hội (Social exchange theory) đã giải thích về việc con người đóng góp cho tổ chức và mong muốn kết quả nhận được từ những đóng góp đó. Có nghĩa là khi con người cho đi một điều gì đó, họ mong nhận lại một điều khác trong tương lai. Nếu một tổ chức thỏa mãn mong muốn của nhân viên, ghi nhận đóng góp của họ và quan tâm đến họ thì người nhân viên sẽ có sự thỏa mãn với công việc, mang lợi ích về cho tổ chức và gia tăng sự hỗ trợ của tổ chức.

Akgunduz, Alkan, và Gök (2018) chứng minh là sự hỗ trợ của tổ chức ảnh hưởng trực tiếp đến sự sáng tạo mà còn ảnh hưởng đến ý nghĩa công việc, và do đó một cách gián tiếp ảnh hưởng đến sự sáng tạo của nhân viên. Vì vậy, sự hỗ trợ của tổ chức có hai tác động thuận chiều, một là tác động lên ý nghĩa công việc, hai là tác động lên sự sáng tạo của nhân viên.

\section{H1: Sự hỗ trợ của tổ chức ảnh hưởng tích cực đến ý nghĩa công việc}

Một số nghiên cứu chứng minh là nhân tố sự hỗ trợ của tổ chức ảnh hưởng trực tiếp đến sự sáng tạo của nhân viên (Akgunduz et al., 2018; Amabile et al., 1996; Dul \& Ceylan, 2011; Horng, Tsai, Yang, Liu, \& Hu, 2016; Ibrahim, Isa, \& Shahbudin, 2016; Joo, Hahn, \& Petersonb, 2015). Nghiên cứu của tác giả Ha và Nguyen (2015) tại trường đại học công lập ở Việt Nam chứng minh là sự hỗ trợ của tổ chức ảnh hưởng tích cực đến sự sáng tạo của nhân viên trường đại học.

\section{H3: Sụ hỗ trợ của tổ chức ảnh hưởng tích cực đến sụ sáng tạo của nhân viên}

\subsection{Tính chủ động cá nhân (Proactive personality, CD)}

Yếu tố chủ động là khả năng sẵn sàng đi trước, tiên phong với những điều mới mẻ, luôn có sáng kiến, giải pháp cho vấn đề gặp phải. Người nhân viên có tính chủ động cá nhân phản ứng chủ động với áp lực của môi trường, thay đổi hoàn cảnh, tìm kiếm thách thức và thành tựu cá nhân. Nghiên cứu của tác giả Akgunduz và cộng sự (2018) đề cập đến tính chủ động của nhân viên, đó là những cá nhân có động lực nội tại mạnh, hành động để đạt được những điều mong muốn, không bị ràng buộc bởi tình huống. Người nhân viên có tính cách chủ động thường thử sắp xếp cuộc sống của họ để đối mặt với thử thách, hoặc thậm chí đổi công việc để tìm kiếm sự 
mạo hiểm. Vì vậy, họ nhận ra ý nghĩa công việc. Tính chủ động cá nhân ảnh hưởng tích cực đến ý nghĩa công việc, đồng thời ý nghĩa công việc làm gia tăng sự sáng tạo của nhân viên. Như vậy, ý nghĩa công việc đóng vai trò trung gian trong mối quan hệ giữa tính chủ động cá nhân ảnh hưởng đến sự sáng tạo của nhân viên.

\section{H2: Tính chủ động cá nhân ảnh hưởng tích cực đến ý nghĩa công việc}

Tính chủ động của nhân viên thể hiện động lực nội tại mạnh, hành động để đạt được những điều mong muốn, không bị ràng buộc bởi tình huống. Nghiên cứu của tác giả Akgunduz và cộng sự (2018) cho rằng nhân viên có tính chủ động cá nhân là những người có động lực nội tại mạnh mẽ. Nghiên cứu của tác giả Horng và cộng sự (2016) và Bateman và Crant (1993) đồng quan điểm, và tìm ra mối quan hệ giữa tính chủ động cá nhân, động lực nội tại và sự sáng tạo của nhân viên. Nghiên cứu của Joo và Lim (2009) tìm hiểu mối quan hệ giữa tính chủ động cá nhân và động lực nội tại, kết quả là những người nhân viên có tính chủ động cá nhân cao thì thường thể hiện động lực nội tại cao. Như vậy, tính chủ động cá nhân có ảnh hưởng tích cực đến động lực nội tại.

\section{H5: Tính chủ động cá nhân ảnh hưởng tích cực đến động lục nội tại}

\section{3. Ý nghĩa công việc (Meaning of work, $Y N$ )}

Ý nghĩa công việc liên quan đến việc tìm kiếm công việc có ý nghĩa, có giá trị và thời gian làm việc bỏ ra là xứng đáng. Ý nghĩa công việc gồm có ba thành phần: ý nghĩa cuộc sống; kinh nghiệm làm việc; định hướng cuộc sống và kế hoạch tương lai của nhân viên; nhận thức của nhân viên về bản thân họ và môi trường xung quanh (Jaiswal \& Dhar, 2015; Spreitzer, 1995). Nghiên cứu của Akgunduz và cộng sự (2018) tổng kết sự sáng tạo phụ thuộc nhiều vào hai nhân tố: sự thỏa mãn với công việc và giá trị bản thân nhận được, đây chính là ý nghĩa công việc. Điều này có nghĩa là ý nghĩa công việc tỷ lệ thuận với sự sáng tạo của nhân viên.

\section{H4: Ý nghĩa công việc ảnh hưởng tích cực đến sụ sáng tạo của nhân viên}

Nói đến yếu tố sự hỗ trợ của tổ chức, đây là yếu tố liên quan đến sự trao quyền tâm lý làm tăng mong muốn của nhân viên và liên quan đến sự sáng tạo của nhân viên. Khi được trao quyền tâm lý, nhân viên cảm thấy công việc quan trọng, có ý nghĩa, ảnh hưởng đến sự sáng tạo. Ngược lại, khi bị tổ chức gò bó, giới hạn, không có sự hỗ trợ thì sẽ không thể đạt được tiềm năng sáng tạo. Sự hỗ trợ của tổ chức khiến nhận viên cảm thấy công việc có ý nghĩa hơn. Điều này dẫn đến nhân viên có hành vi tích cực, làm hài lòng khách hàng, mang thành quả về cho tổ chức (Akgunduz et al., 2018; Coelho, Augusto, \& Lages, 2011). Vì vậy, ý nghĩa công việc không chỉ tác động đến sự sáng tạo của nhân viên mà còn đóng vai trò trung gian trong hai mối quan hệ giữa sự hỗ trợ của tổ chức với sự sáng tạo của nhân viên và tính tự chủ cá nhân đối với sự sáng tạo của nhân viên.

H7a: Ý nghĩa công việc là yếu tố trung gian trong mối quan hệ giữa sư hỗ trợ của tổ chưc và sụ sáng tạo của nhân viên

Hai yếu tố tính chủ động cá nhân và sự hỗ trợ của tổ chức ảnh hưởng cùng chiều với ý nghĩa công việc. Người nhân viên có tính chủ động cá nhân thì có nhiệt huyết cao trong việc giúp xác định cơ hội và đạt thành quả ý nghĩa trong công việc. Tính chủ động cá nhân gia tăng sẽ thúc đẩy sự sáng tạo của nhân viên khi mà nhân viên cảm thấy được ý nghĩa công việc và động lực nội tại (Akgunduz et al., 2018; Coelho et al., 2011; Horng et al., 2016).

H7b: Ý nghĩa công việc là yếu tố trung gian trong mối quan hệ giữa tính chủ động cá nhân và sụ sáng tạo của nhân viên 
H7c: Động lực nội tại là yếu tố trung gian trong mối quan hệ giữa tính chủ động cá nhân và sáng tạo của nhân viên

\section{4. Động lục nội tại (Intrinsic motivation, $D L$ )}

Động lực của con người gồm động lực bên trong (động lực nội tại) và động lực bên ngoài. Động lực nội tại là yếu tố xác định một người nhân viên sẽ thực sự làm/ thực hiện một hoạt động gì đó, họ có khả năng giải quyết vấn đề nảy sinh trong công việc và họ chắc chắn sẽ làm, trong đó động lực nội tại được cho là làm gia tăng sự sáng tạo nhiều hơn hẳn động lực bên ngoài và động lực nội tại là nhân tố quan trọng nhất ảnh hưởng đến sự sáng tạo (Amabile, 1997). Động lực nội tại làm gia tăng đáng kể sự sáng tạo của nhân viên (Horng et al., 2016). Nhân viên có động lực nội tại sẽ thực hiện công việc một cách hào hứng, thích thú hơn và như vậy làm tăng sự sáng tạo (Coelho et al., 2011; Eder \& Sawyer, 2008). Nghiên cứu của tác giả Ha và Nguyen (2015) đề cập đến yếu tố động lực nội tại, và nghiên cứu khẳng định động lực nội tại tác động tích cực đến sự sáng tạo của nhân viên làm việc trong trường đại học Tài chính-Marketing.

\section{H6: Động lực nội tại ảnh hưởng tích cực đến sự sáng tạo của nhân viên}

Tổng hợp và phân tích các công trình nghiên cứu trước đây tác giả xác định giả thuyết và mô hình nghiên cứu như Hình 1. Mô hình nghiên cứu được xây dựng bao gồm 05 yếu tố. Chúng tôi sẽ nghiên cứu ảnh hưởng của sự "hỗ trợ tổ chức" và tính "chủ động cá nhân" ảnh hưởng đến sự sáng tạo của người nhân viên. Hai nhân tố "ý nghĩa công việc" và "động lực nội tại” đóng vai trò là biến trung gian trong các mối quan hệ.

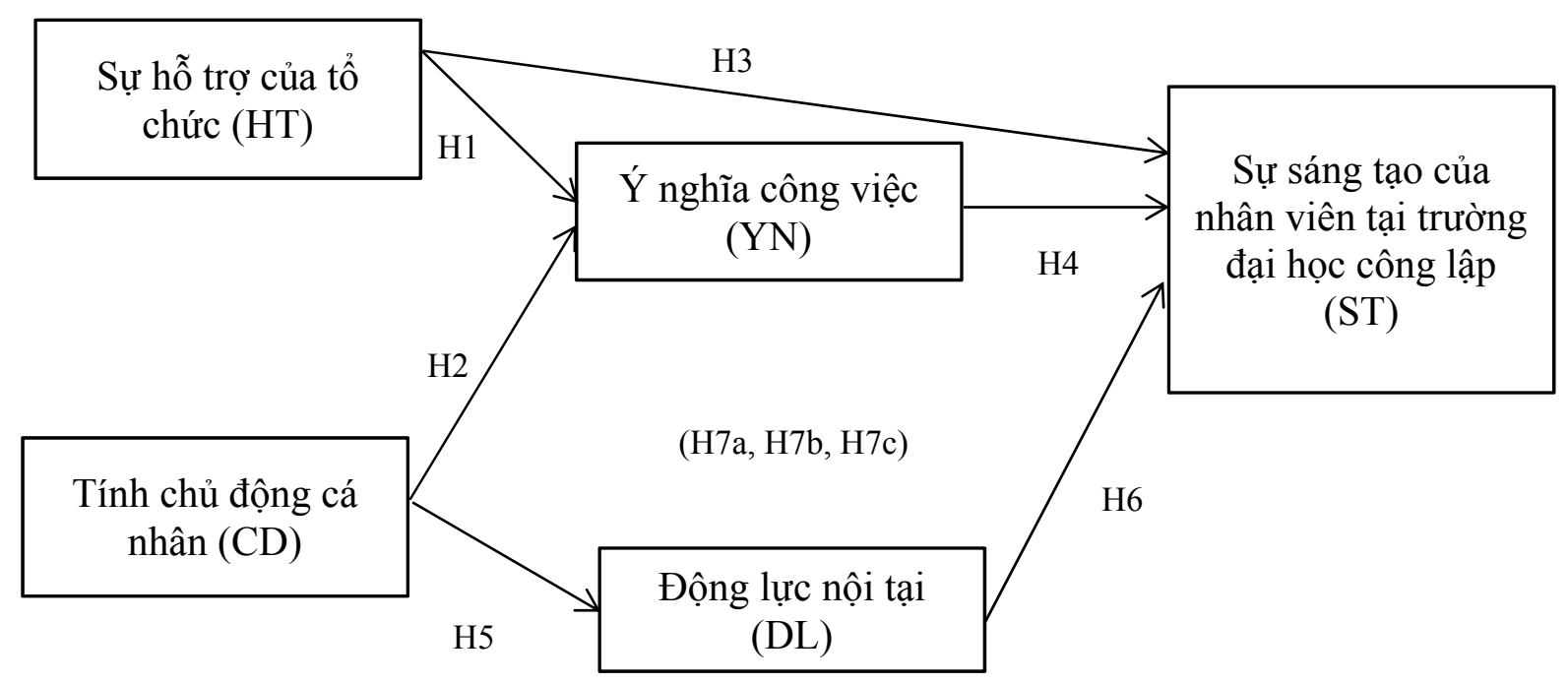

Hình 1. Mô hình nghiên cứu

Các giả thuyết của mô hình được đặt ra như sau:

Giả thuyết 1 (H1): Sự hỗ trợ của tổ chức ảnh hưởng tích cực đến ý nghĩa công việc

Giả thuyết 2 (H2): Tính chủ động cá nhân ảnh hưởng tích cực đến ý nghĩa công việc

Giả thuyết 3 (H3): Sự hỗ trợ của tổ chức ảnh hưởng tích cực đến sự sáng tạo của nhân viên

Giả thuyết 4 (H4): Ý nghĩa công việc ảnh hưởng tích cực đến sự sáng tạo của nhân viên.

Giả thuyết 5 (H5): Tính chủ động cá nhân ảnh hưởng tích cực đến động lực nội tại

Giả thuyết 6 (H6): Động lực nội tại ảnh hưởng tích cực đến sự sáng tạo của nhân viên

Giả thuyết $7 \mathrm{a}(\mathrm{H} 7 \mathrm{a})$ : Ý nghĩa công việc là yếu tố trung gian trong mối quan hệ giữa sự hỗ trợ của tổ chức và sự sáng tạo của nhân viên

Giả thuyết $7 \mathrm{~b}(\mathrm{H} 7 \mathrm{~b})$ : Ý nghĩa công việc là yếu tố trung gian trong mối quan hệ giữa tính chủ động cá nhân và sự sáng tạo của nhân viên

Giả thuyết 7c $(\mathrm{H} 7 \mathrm{c})$ : Động lực nội tại là yếu tố trung gian trong mối quan hệ giữa tính chủ động cá nhân và sự sáng tạo của nhân viên 


\section{Phương pháp nghiên cứu}

\subsection{Cõ̃ mẫu phân tích và thu thập số liệu}

Nghiên cứu tác giả Hair, Black, Babin, Anderson, và Tatham (2006) cho rằng mẫu nghiên cứu phải đạt kích thước tối thiểu là 50 và có tỷ lệ quan sát so với biến đo lường là $5: 1$ đối với phương pháp phân tích nhân tố khám phá (EFA). Bài nghiên cứu này có 28 biến đo lường nên kích thước mẫu là $28 \times 5=140$. Đối với mô hình cấu trúc tuyến tính (SEM) thì không có công thức cụ thể để tính cỡ mẫu như phân tích EFA. Tuy nhiên, tác giả Kline (2011) cho rằng cỡ mẫu hợp lý phải đạt được để mô hình SEM xử lý ổn định là 200 nên bài nghiên cứu đặt mục thu thập tiêu tối thiểu 200 bản trả lời khảo sát. Đối tượng khảo sát là các nhân viên có thâm niên làm việc trên 05 năm tại các phòng chức năng như: tổ chức - hành chính, đào tạo, công tác sinh viên, quản lý khoa học, đối ngoại, văn phòng tại 09 trường đại học công lập tại Thành phố Hồ Chí Minh. Do điều kiện khách quan, nhóm tác giả sử dụng phương pháp chọn mẫu thuận tiện, với hình thức gửi bản hỏi trực tuyến qua mail cá nhân. Khảo sát được tiến hành từ tháng 08 đến tháng 12 năm 2019. Tiêu chí chọn mẫu là những trường đại học công lập lớn, trên địa bàn Thành phố Hồ Chí Minh, có sự quản lý của Bộ Giáo dục và Đào tạo để đảm bảo sự tương đồng về cơ chế quản lý. Thứ hai, ngành nghề đào tạo của trường đại học này bao phủ đa số lĩnh vực phổ biến được đào tạo tại Thành phố Hồ Chí Minh, bao gồm: trường Đại học Bách khoa, trường Đại học Khoa học Tự nhiên, trường Đại học Khoa học xã hội \& Nhân văn, trường Đại học Kinh tế Luật, trường Đại học Quốc tế, trường Đại học Công nghệ thông tin, trường Đại học Sư phạm Kỹ thuật, Cơ quan Đại học Quốc gia Thành phố Hồ Chí Minh và Khoa Y Đại học Quốc gia Thành phố Hồ Chí Minh.

Kết quả khảo sát nhận được 256 bản trả lời, trong đó có 247 bản trả lời hợp lệ, đạt tỷ lệ $96,48 \%$ (Bảng 1). Phần lớn người thực hiện khảo sát làm việc ở các vị trí nhân viên (chiếm 75\%), là nữ (chiếm 64\%), độ tuổi khá trẻ (độ tuổi 31- 40 tuổi chiếm tỷ lệ 57\%), trình độ đại học (chiếm 55\%) và thạc sĩ (chiếm 43\%), mức thu nhập trung bình hạn chế (mức 8 - 14 triệu/ tháng chiếm tỷ lệ $51 \%$ và dưới 8 triệu/ tháng chiếm tỷ lệ $36 \%$ ).

\section{Bảng 1}

Mô tả đặc điểm nhân khẩu học của đối tượng khảo sát

\begin{tabular}{|l|c|c|}
\hline \multicolumn{1}{|c|}{ Nội dung } & Số lượng & Tỷ lệ \% \\
\hline Đơn vị làm việc: & $\mathbf{2 4 7}$ & $\mathbf{1 0 0}$ \\
Trường Đại học Bách khoa & 29 & 11.7 \\
Trường Đại học Khoa học Tự nhiên & 35 & 14.2 \\
Trường Đại học Khoa học xã hội \& Nhân văn & 28 & 11.3 \\
Trường Đại học Kinh tế - Luật & 33 & 13.3 \\
Trường Đại học Quốc tế & 18 & 7.3 \\
Trường Đại học Công nghệ thông tin & 16 & 6.5 \\
Trường Đại học Sư phạm Kỹ thuật & 14 & 5.7 \\
Cơ quan Đại học Quốc gia Thành phố Hồ Chí Minh & 57 & 23.1 \\
Khoa Y Đại học Quốc gia Thành phố Hồ Chí Minh & 17 & 6.9 \\
\hline Vị trí công việc: & $\mathbf{2 4 7}$ & $\mathbf{1 0 0}$ \\
Nhân viên & 150 & 74.9 \\
Trưởng/ Phó phòng & 50 & 20.2 \\
Lãnh đạo đơn vị (cấp trên Trưởng phòng) & 12 & 4.9 \\
\hline
\end{tabular}




\begin{tabular}{|l|c|c|}
\hline \multicolumn{1}{|c|}{ Nội dung } & Số lượng & Tỷ lệ \% \\
\hline Giới tính: & $\mathbf{2 4 7}$ & $\mathbf{1 0 0}$ \\
Nam & 88 & 35.6 \\
Nữ & 159 & 64.4 \\
\hline Tuổi: & $\mathbf{2 4 7}$ & $\mathbf{1 0 0}$ \\
22 - 30 tuổi & 64 & 25.9 \\
31 - 40 tuổi & 142 & 57.5 \\
41 - 50 tuổi & 38 & 15.4 \\
Trên 50 tuổi & 3 & 1.2 \\
\hline Trình độ học vấn: & $\mathbf{2 4 7}$ & $\mathbf{1 0 0}$ \\
Cao đẳng & 1 & 0.4 \\
Đại học & 135 & 54.7 \\
Thạ́c sĩ & 107 & 43.3 \\
Tiến sĩ & 2 & 0.8 \\
Trình độ khác & 2 & 0.8 \\
\hline Thu nhập trung bình/ tháng: & $\mathbf{2 4 7}$ & $\mathbf{1 0 0}$ \\
Dưới 8 triệu & 88 & 35.6 \\
Từ 8 đến dưới 14 triệu & 126 & 51.0 \\
Từ 14 đến dưới 20 triệu & 26 & 10.5 \\
Từ 20 triệu trở lên & 7 & 2.8 \\
\hline Thâm niên làm việc: & $\mathbf{2 4 7}$ & $\mathbf{1 0 0}$ \\
Dưới 5 năm & 57 & 23.1 \\
Từ 5 đến dưới 10 năm & 65 & 26.3 \\
Từ 10 đến dưới 15 năm & 69 & 27.9 \\
Từ 15 đến dưới 20 năm & 23 & 9.3 \\
\hline Từ 20 năm trở lên & 33.4 \\
\hline
\end{tabular}

Nguồn: Tính toán của tác giả từ số liệu điều tra nhân viên tại các trường đại học công lập ở Thành phố Hồ Chí Minh năm 2019

\subsection{Thang đo các khái niệm nghiên cúu}

Bảng câu hỏi gồm hai phần khảo sát chính. Phần thứ nhất thu thập thông tin cá nhân giúp hiểu rõ hơn về đối tượng khảo sát: giới tính, độ tuổi, thâm niên làm việc, thu nhập tháng, chức vụ/ vị trí công việc. Độ tuổi và thu nhập trả lời bằng hình thức chọn khoảng số liệu. Từ đó có cái nhìn bao quát về mẫu, giúp phần nhận định kết quả được rõ ràng hơn.

Phần thứ hai của thang đo là khảo sát về sự sáng tạo của nhân viên và các yếu tố ảnh hưởng đến sự sáng tạo của nhân viên, trong đó có 05 nhóm câu hỏi : (1) Sự sáng tạo của nhân viên (kí hiệu là $\mathrm{ST}$ ) được đo lường bởi 04 biến quan sát $(\alpha=0.842)$; (2) Sự hỗ trợ của tổ chức (kí hiệu là HT) được đo lường bởi 08 biến quan sát $(\alpha=0.922)$; (3) Tính chủ động cá nhân (kí hiệu là $\mathrm{CD}$ ) được đo lường bởi 10 biến quan sát $(\alpha=0.914)$; (4) Ý nghĩa công việc (kí hiệu là YN) với 03 biến quan sát $(\alpha=0.887$ ); (5) Động lực nội tại (ký hiệu là DL) với 03 biến quan sát $(\alpha=$ 0.803). Phần khảo sát gồm có 28 câu hỏi được trình bày như Bảng 2 . Thang đo sử dụng Likert 5 mức độ với mức điểm 1 đến 5 là hoàn toàn không đồng ý, không đồng ý, trung lập, đồng ý và hoàn toàn đồng ý. 
Để xây dựng thang đo cho các biến sử dụng trong bài nghiên cứu, tác giả tham khảo từ các nghiên cứu liên quan, có trình bày ở Phần 2 . Đầu tiên, tác giả tham khảo thang đo từ những công trình nghiên cứu liên quan, dịch sang tiếng Việt và tác giả có thang đo nháp. Tiếp theo, một phương pháp nghiên cứu định tính được thực hiện là công cụ phỏng vấn sâu để điều chỉnh thang đo cho phù hợp với đối tượng khảo sát, môi trường đặc thù của trường đại học công lập. Việc phỏng vấn ở đây là phỏng vấn tay đôi nhằm điều chỉnh ngôn ngữ, cách diễn đạt thang đo khi dịch từ bản tiếng Anh hoặc bổ sung thông tin cần thêm cho thang đo (nếu có). Phỏng vấn tay đôi dễ áp dụng hơn vì rất khó sắp xếp việc thảo luận nhóm và đa số đối tượng được phỏng vấn có chức vụ quản lý nên cuộc phỏng vấn mang ý kiến cá nhân, riêng tư, không phù hợp trong thảo luận nhóm. Ngoài ra, tác giả nhận thấy việc phỏng vấn tay đôi dễ khai thác những quan niệm, suy nghĩ sâu xa của người được phỏng vấn hơn thảo luận nhóm. Phỏng vấn tay đôi nhằm giúp tiếp cận đối tượng nhanh hơn, người được phỏng vấn cởi mở hơn và tác giả dễ làm cho họ hiểu được nội dung cần phỏng vấn.

Việc phỏng vấn tay đôi được thực hiện lần lượt từ cấp nhân viên (không có chức vụ quản lý), đến quản lý cấp trung (Trưởng phòng) và quản lý cấp cao. Tất cả những người được phỏng vấn đều có thâm niên trên 05 năm và rất $\mathrm{am}$ hiểu về kiến thức trong ngành giáo dục cũng như kinh nghiệm quản lý nhân sự đối với cấp quản lý. Lần lượt từng thang đo được hình thành:

+ Sự sáng tạo của nhân viên: có 02 thang đo tham khảo của Jaiswal và Dhar (2015) với 04 biến quan sát và của Zhou và George (2001) với 13 biến quan sát. Kết quả qua các cuộc phỏng vấn đều nghiên về thang đo của Jaiswal và Dhar (2015) vì mới hơn, dễ hiểu, không có những câu hỏi trùng ý và dễ gây hiểu lầm như thang đo còn lại. Ngoài ra đây cũng là thang đo được sử dụng trong nghiên cứu của Akgunduz và cộng sự (2018), là nghiên cứu nền chính của bài nghiên cứu của tác giả;

+ Đối với thang đo của các biến còn lại, tác giả đều dùng thang đo trong các công trình nghiên cứu trước đây, không thêm bớt biến, chỉ điều chỉnh cách diễn đạt các biến cho phù hợp đối tượng khảo sát và đảm bảo không làm thay đổi ý nghĩa thang đo khi chuyển từ tiếng Anh sang tiếng Việt. Nhìn chung, từng biến quan sát trong từng thang đo đều được đưa ra phân tích, điều chỉnh phù hợp, đảm bảo dịch đúng nghĩa đồng thời phải dễ hiểu đối với đối tượng khảo sát.

\section{Bảng 2}

Câu hỏi khảo sát sử dụng trong nghiên cứu và nguồn tham khảo

\begin{tabular}{|c|c|c|c|}
\hline Nhân tố & Tiêu chí đo & $\begin{array}{c}\text { Kí } \\
\text { hiệu }\end{array}$ & $\begin{array}{l}\text { Nguồn tham } \\
\text { khảo }\end{array}$ \\
\hline \multirow{4}{*}{$\begin{array}{l}\text { Sự sáng tạo } \\
\text { của nhân } \\
\text { viên }(\mathrm{ST})\end{array}$} & Anh chị nhận ra cơ hội cho những cách giải quyết công việc mới & ST1 & \multirow{4}{*}{$\begin{array}{c}\text { Jaiswal và } \\
\text { Dhar (2015) }\end{array}$} \\
\hline & Anh chị tìm kiếm ý tưởng mới/ cách mới để giải quyết vấn đề & ST2 & \\
\hline & Anh chị tạo ra ý tưởng mới lạ nhưng có thể thực hiện được & ST3 & \\
\hline & Anh chị thể hiện sự độc đáo trong công việc của mình & ST4 & \\
\hline \multirow{4}{*}{$\begin{array}{l}\text { Sự hỗ trợ } \\
\text { của tồ chức } \\
(\mathrm{HT})\end{array}$} & $\begin{array}{l}\text { Tổ chức đánh giá cao sự đóng góp của anh chị cho sự thịnh } \\
\text { vượng của tồ chức }\end{array}$ & HT1 & \multirow{4}{*}{$\begin{array}{l}\text { Eisenberger } \\
\text { và cộng sự } \\
\text { (1986) }\end{array}$} \\
\hline & $\begin{array}{l}\text { Tổ chức không đánh giá cao bất kỳ sự cố gắng THÊM (extra } \\
\text { effort) nào của anh chị }\end{array}$ & HT2 & \\
\hline & Tổ chức bỏ qua những ý kiến, phản ánh của anh chị & HT3 & \\
\hline & Tổ chức thực sự quan tâm đến sự vui vẻ của anh chị & HT4 & \\
\hline
\end{tabular}




\begin{tabular}{|c|c|c|c|}
\hline Nhân tố & Tiêu chí đo & $\begin{array}{c}\text { Kí } \\
\text { hiệu }\end{array}$ & $\begin{array}{l}\text { Nguồn tham } \\
\text { khảo }\end{array}$ \\
\hline & $\begin{array}{l}\text { Ngay cả khi anh chị làm việc tốt nhất có thể thì tổ chức cũng } \\
\text { không chú ý }\end{array}$ & HT5 & \\
\hline & Tổ chức quan tâm đến sự hài lòng trong công việc của anh chị & HT6 & \\
\hline & Tổ chức rất ít quan tâm đến anh chị & HT7 & \\
\hline & Tổ chức tự hào về thành tích của anh chị trong công việc & HT8 & \\
\hline \multirow{10}{*}{$\begin{array}{l}\text { Tính chủ } \\
\text { động cá } \\
\text { nhân (CD) }\end{array}$} & $\begin{array}{l}\text { Anh chị luôn luôn tìm kiếm những cách mới để cải thiện } \\
\text { cuộc sông của mình }\end{array}$ & $\mathrm{CD} 1$ & \multirow{10}{*}{$\begin{array}{l}\text { Bateman và } \\
\text { Crant (1993) }\end{array}$} \\
\hline & $\begin{array}{l}\text { Bất cứ khi nào, anh chị đều có động lực mạnh mẽ cho sự } \\
\text { thay đổi tích cực }\end{array}$ & $\mathrm{CD} 2$ & \\
\hline & $\begin{array}{l}\text { Không có gì thú vị hơn việc nhìn thấy ý tưởng của anh chị } \\
\text { trở thành hiện thực }\end{array}$ & CD3 & \\
\hline & Nếu nhìn thấy điều gì không thích, anh chị sẽ thay đổi nó & CD4 & \\
\hline & $\begin{array}{l}\text { Với bất kỳ tỷ lệ thành công nào, nếu anh chị tin điều gì đó, } \\
\text { anh chị sẽ làm nó xảy ra }\end{array}$ & CD5 & \\
\hline & $\begin{array}{l}\text { Anh chị thích những ý tưởng của mình trở thành xuất sắc } \\
\text { nhất, dù cho phải chống lại những ý kiến phản đối }\end{array}$ & CD6 & \\
\hline & Anh chị xuất sắc trong việc nhận ra những cơ hội & $\mathrm{CD} 7$ & \\
\hline & Anh chị luôn luôn tìm kiếm những cách tốt hơn để làm việc & CD8 & \\
\hline & $\begin{array}{l}\text { Nếu anh chị tin vào một ý tưởng thì không có trở ngại nào có } \\
\text { thể ngăn anh chị thực hiện nó }\end{array}$ & CD9 & \\
\hline & $\begin{array}{l}\text { Anh chị có thể nhận ra cơ hội tốt từ lâu trước khi những } \\
\text { người khác có thể nhận ra }\end{array}$ & $\mathrm{CD} 10$ & \\
\hline \multirow{3}{*}{$\begin{array}{l}\text { Ý nghĩa } \\
\text { công việc } \\
\text { (YN) }\end{array}$} & Công việc anh chị đang làm rất quan trọng với anh chị & YN1 & \multirow{3}{*}{$\begin{array}{l}\text { Spreitzer } \\
(1995)\end{array}$} \\
\hline & Các hoạt động trong công việc có ý nghĩa với cá nhân anh chị & YN2 & \\
\hline & $\begin{array}{l}\text { Anh chị đang làm việc, thực hiện nhiệm vụ công việc có ý } \\
\text { nghĩa với anh chị }\end{array}$ & YN3 & \\
\hline \multirow{3}{*}{$\begin{array}{l}\text { Động lực } \\
\text { (DL) nội tại }\end{array}$} & Anh chị thích tìm giải pháp cho những vấn đề phức tạp & DL1 & \multirow{3}{*}{$\begin{array}{c}\text { Zhang và } \\
\text { Bartol (2010) }\end{array}$} \\
\hline & Anh chị thích thiết lập các quy trình làm việc & DL2 & \\
\hline & Anh chị thích cải thiện quy trình/ sản phẩm hiện có & DL3 & \\
\hline
\end{tabular}

Nguồn: Kết quả xử lý dữ liệu của nhóm tác giả

\subsection{Phương pháp phân tích}

Tác giả sử dụng hệ số Cronbach's Alpha để loại bỏ các biến có độ tin cậy thang đo thấp (T. D. Nguyen, 2011, 2014). Kết quả nghiên cứu cho thấy, các biến đều đạt tiêu chuẩn về độ tin cậy thang đo.

Phân tích nhân tố khẳng định (Confirmatory finding Analysis, CFA) đã được sử dụng để kiểm tra độ tin cậy cấu trúc của mô hình đo lường (construct validity of the measuremen model) trong khi mô hình phương trình cấu trúc (SEM) đã được sử dụng để kiểm tra các giả thuyết và tính hợp lệ của mô hình nghiên cứu (Anderson \& Gerbing, 1988). 
Mô hình phương trình cấu trúc tuyến tính dựa trên hiệp phương sai (CB-SEM) được sử dụng để phân tích dữ liệu và các kiểm định mối quan hệ của giả thuyết (Baumgartner \& Homburg, 1996; Hair et al., 2006; Kline, 2011). Tác giả sử dụng phần mềm IBM AMOS (v. 20.0.0, 2011, New York, NY, USA) để thực hiện phân tích CB-SEM.

Ở bước đầu tiên, mô hình đo lường đã được áp dụng kiểm tra tính hợp lệ và độ tin cậy của các thang đo. Độ tin cậy (reliability) của các biến quan sát phải có hệ số tải nhân tố (outer loadings) lớn hơn hoặc bằng 0.5 thì đạt yêu cầu về độ tin cậy và hệ số tin cậy tổng hợp (composite reliability, CR) phải lớn hơn hoặc bằng 0.7 thì đạt độ tin cậy tổng hợp (Hulland, 1999). Giá trị hội tụ (convergent validity) được sử dụng để đánh giá sự ổn định của thang đo. Theo tác giả Hair, Black, Babin, và Anderson (2010), hệ số phương sai trung bình trích (average variance extracted, AVE) phải lớn hơn hoặc bằng 0.5 khẳng định được mức độ giá trị hội tụ. Giá trị phân biệt (discriminant validity) giúp đảm bảo sự khác biệt, không có mối tương quan giữa các yếu tố. Để đo lường giá trị phân biệt thì căn bậc hai AVE (SQRTAVE) của mỗi nhân tố đo lường đều lớn hơn hệ số tương quan (latent variable correlations/Inter-Construct Correlations) giữa nhân tố đó với các nhân tố khác cho thấy giá trị phân biệt và mức độ tin cậy của các nhân tố.

Kế tiếp, mô hình CB-SEM được xác định để kiểm định mối quan hệ của các giả thuyết nghiên cứu. Nếu kết quả có giá trị t-value $>1.96$ thì kiểm định có ý nghĩa thống kê ở mức 5\%.

\section{Kết quả phân tích}

\subsection{Kiểm định mô hình đo lưòng (measurement model)}

Kết quả kiểm định độ tin cậy bằng hệ số Cronbach's Alpha cho thấy hệ số Cronbach's Alpha trong khoảng 0.6 - 0.9 và không có biến đo lường nào có tương quan bé hơn 0.3 ; trong đó hệ số $\alpha(\mathrm{ST})=0.842, \alpha(\mathrm{HT})=0.922, \alpha(\mathrm{CD})=0.914, \alpha(\mathrm{DL})=0.803, \alpha(\mathrm{YN})=0.887$. Hệ số tương quan biến tổng hiệu chỉnh đều lớn hơn 0.3 và hệ số $\alpha$ khi loại bỏ từng biến quan sát đều không tốt hơn. Như vậy, hệ số Cronbach's Alpha của các thang đo đạt yêu cầu và 28 biến quan sát tiếp tục được đưa vào phân tích EFA.

\subsubsection{Phân tích nhân tố khám phá (EFA)}

Sau khi thang đo các nhân tố đạt kiểm định hệ số tin cậy Cronbach’s Alpha với 28 biến đều đạt yêu cầu, nhóm tác giả tiến hành phân tích EFA. Phương pháp trích nhân tố sử dụng phương pháp phép xoay Promax. Thang đo được chấp nhận khi $0.5<$ Kaiser-Meyer-Olkin $(\mathrm{KMO})<1$, và hệ số $\mathrm{Sig} .=0.000$ của kiểm định Bartlett cho biết các biến quan sát tương quan với nhau có ý nghĩa thống kê (Kline, 2011). Tổng phương sai trích có giá trị $>50 \%$ và hệ số tải nhân tố > 0.5 chứng tỏ đạt độ tin cậy thích hợp cho phân tích EFA (Anderson \& Gerbing, 1988). Bảng 3 thể hiện hệ số tải nhân tố của các biến quan sát ở năm nhân tố đều lớn 0.5 . Chênh lệch hệ số tải nhân tố ở từng biến quan sát đều lớn hơn 0.3 đảm bảo đạt yêu cầu. Như vậy, thang đo đã được kiểm định đạt giá trị hội tụ và phân biệt.

Kết quả phân tích EFA dừng lại ở lần xoay nhân tố thứ 05 với chỉ số KMO bằng 0.913 và giá trị kiểm định Bartlett có ý nghĩa với Sig. $=0.000$ chứng tỏ các biến quan sát có tương quan với nhau xét trên phạm vi tổng số quan sát. Kết quả phương pháp phân tích EFA trích được 05 nhân tố với 28 biến quan sát, bằng số lượng khái niệm nghiên cứu đưa ra trong phần lý thuyết. Thang đo giá trị Eigenvalue bằng 1.119 và tổng phương sai trích (cumulative total variance explained) là $59.490 \%>50 \%$. Điều này có nghĩa là tại hệ số điểm dừng là 1.119 thì 05 nhân tố này giải thích được $59.490 \%$ độ biến thiên của dữ liệu. 


\section{Bảng 3}

Kết quả ma trận trọng số nhân tố ảnh hưởng đến sự sáng tạo và kết quả độ tin cậy thang đo

\begin{tabular}{|c|c|c|c|c|c|}
\hline \multirow{2}{*}{ Biến quan sát } & \multicolumn{5}{|c|}{ Nhân tố } \\
\hline & Chủ động & Hỗ trọ" & Sáng tạo & Ý nghĩa & Động lực \\
\hline ST1 & & & 0.790 & & \\
\hline ST2 & & & 0.740 & & \\
\hline ST3 & & & 0.702 & & \\
\hline ST4 & & & 0.568 & & \\
\hline HT1 & & 0.683 & & & \\
\hline HT2 & & 0.780 & & & \\
\hline HT3 & & 0.856 & & & \\
\hline HT4 & & 0.677 & & & \\
\hline HT5 & & 0.863 & & & \\
\hline HT6 & & 0.735 & & & \\
\hline HT7 & & 0.866 & & & \\
\hline HT8 & & 0.703 & & & \\
\hline CD1 & 0.745 & & & & \\
\hline $\mathrm{CD} 2$ & 0.733 & & & & \\
\hline CD3 & 0.725 & & & & \\
\hline $\mathrm{CD} 4$ & 0.683 & & & & \\
\hline CD5 & 0.615 & & & & \\
\hline CD6 & 0.756 & & & & \\
\hline CD7 & 0.713 & & & & \\
\hline $\mathrm{CD} 8$ & 0.747 & & & & \\
\hline CD9 & 0.719 & & & & \\
\hline CD10 & 0.710 & & & & \\
\hline DL1 & & & & & 0.581 \\
\hline DL2 & & & & & 0.895 \\
\hline DL3 & & & & & 0.717 \\
\hline YN1 & & & & 0.799 & \\
\hline YN2 & & & & 0.803 & \\
\hline YN3 & & & & 0.930 & \\
\hline Eigen value & 9.088 & 5.111 & 1.899 & 1.349 & 1.119 \\
\hline Cronbach's Alpha & 0.842 & 0.922 & 0.914 & 0.803 & 0.887 \\
\hline Tổng phương sai trích (\%) & 31.033 & 47.892 & 53.187 & 56.910 & 59.490 \\
\hline
\end{tabular}

Nguồn: Tính toán của tác giả từ số liệu điều tra nhân viên tại các trường đại học công lập ở Thành phố Hồ Chí Minh năm 2019 


\subsubsection{Phân tích nhân tố khẳng định (CFA)}

Hình 2 cho thấy là giá trị Chi-square value là $670.771(\mathrm{df}=340$; RMSEA Root Mean Squared Error of Approximation là 0.063). Chỉ số RMSEA < 0.05 thường được sử dụng làm tiêu chí lựa chọn cho mô hình phù hợp, tuy nhiên, nghiên cứu của MacCallum, Browne, và Sugawara (1996) cho rằng $0.01 ; 0.05$ và 0.08 là tương ứng với sự phù hợp rất tốt, tốt và trung bình. Chỉ số $\mathrm{CMIN/df} \mathrm{có} \mathrm{giá} \mathrm{trị} \mathrm{càng} \mathrm{nhỏ} \mathrm{càng} \mathrm{tốt,} \mathrm{thể} \mathrm{hiện} \mathrm{sự} \mathrm{phù} \mathrm{hợp} \mathrm{của} \mathrm{mô} \mathrm{hình.} \mathrm{Chỉ} \mathrm{số}$ $\mathrm{CMIN} / \mathrm{df}=1.973$ khá nhỏ và $<3$, thể hiện sự phù hợp của mô hình. Một số chỉ số đo lường mức độ phù hợp của mô hình là: chỉ số CFI (Comparative Fit Index) $=0.919$. Tương tự CFI, chỉ số TLI (Tucker Lewis Index) $=0.910$ thường có giá trị tương đương với chỉ số CFI. Chỉ số GFI $($ Goodness Fit Index $)=0.827$. Ba chỉ số CFI, TLI, GFI lớn hơn 0.8 ; có nghĩa là các giá trị này cho thấy mô hình phù hợp.

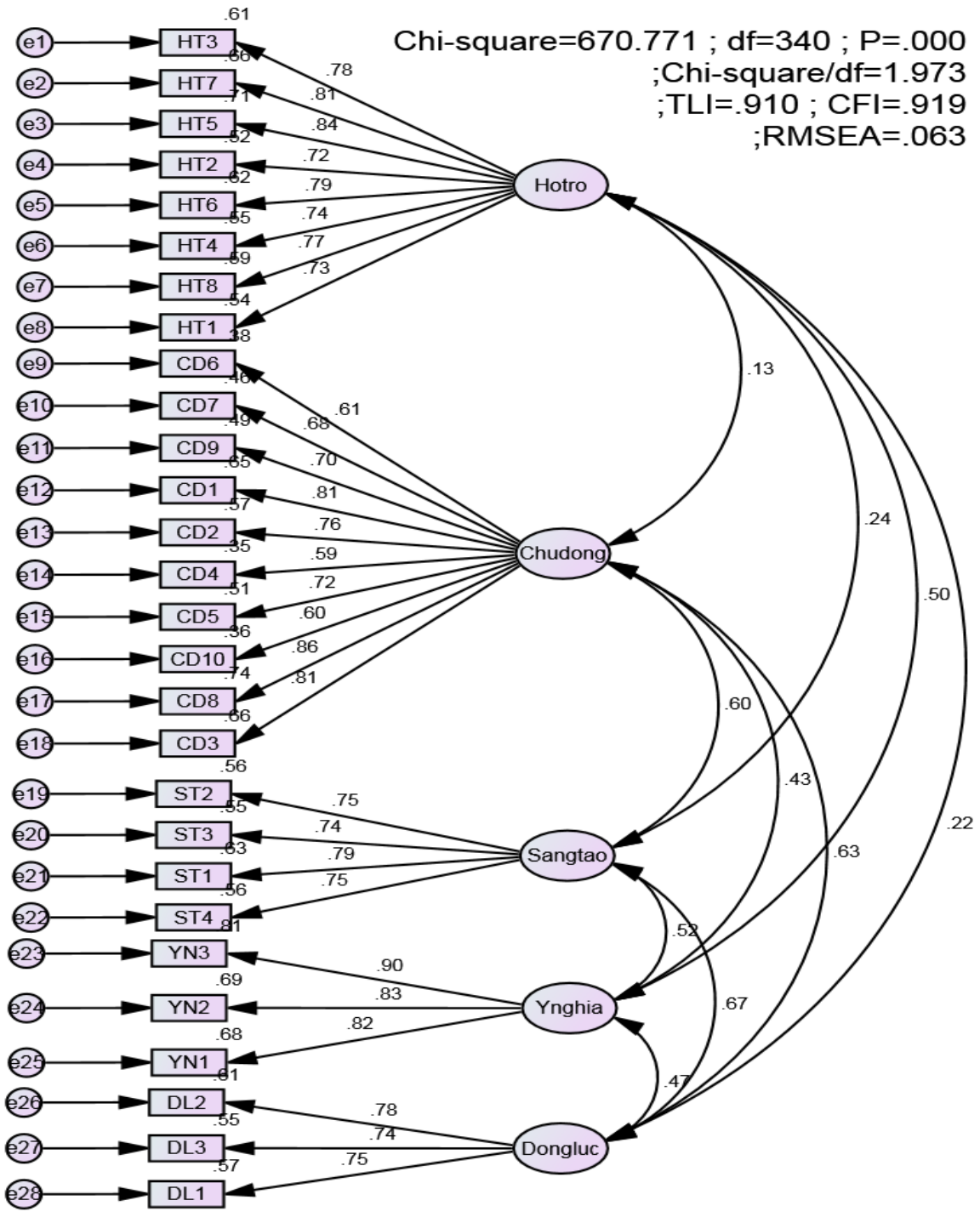

Hình 2. Mô hình phân tích nhân tố khẳng định (CFA)

Đối với phân tích CFA nghiên cứu sử dụng hệ số tin cậy tổng hợp CR, hệ số AVE và hệ số tải chuẩn hóa đánh giá độ tin cậy của thang đo (Asyraf \& Afthanorhan, 2013; Hair, Gabriel, \& Patel, 2014). Bảng 4 cho thấy hệ số $C R$ trong khoảng $0.8-0.9$ và hệ số tải chuẩn hóa lớn hơn 0.4. Ngoài ra, giá trị $\mathrm{AVE}$ của từng thành phần lớn hơn mức 0.5 . Kết quả tính toán về hệ số $\mathrm{CR}$, hệ số tải nhân tố và phương sai trích của các thang đo thành phần cho thấy các thang đo của khái niệm đều đạt yêu cầu về độ tin cậy và giá trị hội tụ. 


\section{Bảng 4}

Giá trị trung bình, độ tin cậy và tương quan

\begin{tabular}{|c|c|c|c|c|c|c|c|c|c|}
\hline $\mathbf{0}$ & Mean & CR & AVE & MSV & $\begin{array}{c}\text { Ý nghĩa } \\
\text { công } \\
\text { việc }\end{array}$ & $\begin{array}{l}\text { Sự hố } \\
\text { trơ của } \\
\text { tổ chức }\end{array}$ & $\begin{array}{c}\text { Tính chủ } \\
\text { động cá } \\
\text { nhân }\end{array}$ & $\begin{array}{c}\text { Sự sáng } \\
\text { tạo của } \\
\text { nhân } \\
\text { viên }\end{array}$ & $\begin{array}{l}\text { Động } \\
\text { lụ̣c nội } \\
\text { tại }\end{array}$ \\
\hline $\begin{array}{l}\text { Ý nghĩa } \\
\text { công việc }\end{array}$ & 3.893 & 0.913 & 0.727 & 0.276 & 0.853 & & & & \\
\hline $\begin{array}{l}\text { Sự hỗ trọ } \\
\text { của tổ } \\
\text { chức }\end{array}$ & 3.686 & 0.923 & 0.600 & 0.247 & $0.497 * *$ & 0.775 & & & \\
\hline $\begin{array}{l}\text { Tính chủ } \\
\text { động cá } \\
\text { nhân }\end{array}$ & 3.748 & 0.843 & 0.517 & 0.393 & $0.426 * *$ & $0.129 * *$ & 0.719 & & \\
\hline $\begin{array}{l}\text { Sự sáng } \\
\text { tạo của } \\
\text { nhân } \\
\text { viên }\end{array}$ & 3.860 & 0.889 & 0.573 & 0.453 & $0.525 * *$ & $0.239 * *$ & $0.601 * *$ & 0.757 & \\
\hline $\begin{array}{l}\text { Động lực } \\
\text { nội tại }\end{array}$ & 3.864 & 0.805 & 0.579 & 0.453 & $0.471 * *$ & $0.220 * *$ & $0.627 * *$ & $0 . .673 * *$ & 0.761 \\
\hline
\end{tabular}

$\sqrt{A V E}$ là các giá trị trên đường chéo được in đậm, chỉ số tương quan giữa hai nhân tố là các giá trị trong ô được tô màu. Có thể thấy $\sqrt{A V E}$ lớn hơn các chỉ số tương quan giữa hai nhân tố; **p $<0.01 ; \mathrm{SD}=$ Standard Deviation

Ghi chú: *; **; *** lần lượt tương ứng với các mức ý nghĩa thống kê $10 \%, 5 \%$ và $1 \%$

Nguồn: Kết quả nghiên cứu định tính và tính toán của nhóm tác giả

\subsection{Kiểm định mô hìh cấu trúc (Structural model)}

Hình 3 cho thấy hệ số ước tính chuẩn hóa của các mối quan hệ của các nhân tố theo giả định. Kết quả mô hình SEM cho thấy giá trị thống kê Chi-bình phương cho thấy sự phù hợp mô hình với dữ liệu nghiên cứu $(\chi 2=691.34 ; \mathrm{df}=343, \mathrm{p}<0.0001 ; \mathrm{X} 2 / \mathrm{df}=2.017$; GFI = 0.823; CFI $=0.915 ; \mathrm{RMSEA}=0.064)$. Giá trị Chi-square value là $691.937(\mathrm{df}=343 ; \mathrm{p}=0.000)$. Chỉ số $\mathrm{CMIN} / \mathrm{df}=2.017$ khá nhỏ và $<3$, thể hiện sự phù hợp của mô hình. Một số các chỉ số đo lường sự phù hợp mô hình là: chỉ số $\mathrm{CFI}=0.915$. Chỉ số $\mathrm{GFI}=0.823$. Hai chỉ số $\mathrm{CFI}$, GFI đều lớn hơn 0.8 ; có nghĩa là các giá trị này cho thấy mô hình phù hợp. Mô hình có giá trị $\mathrm{R}^{2}=59.5 \%$ có nghĩa là các nhân tố sự hỗ trợ tổ chức, chủ động cá nhân, động lực nội tại, ý nghĩa công việc giải thích được 59.50\% sự biến thiên mức độ sự sáng tạo. 


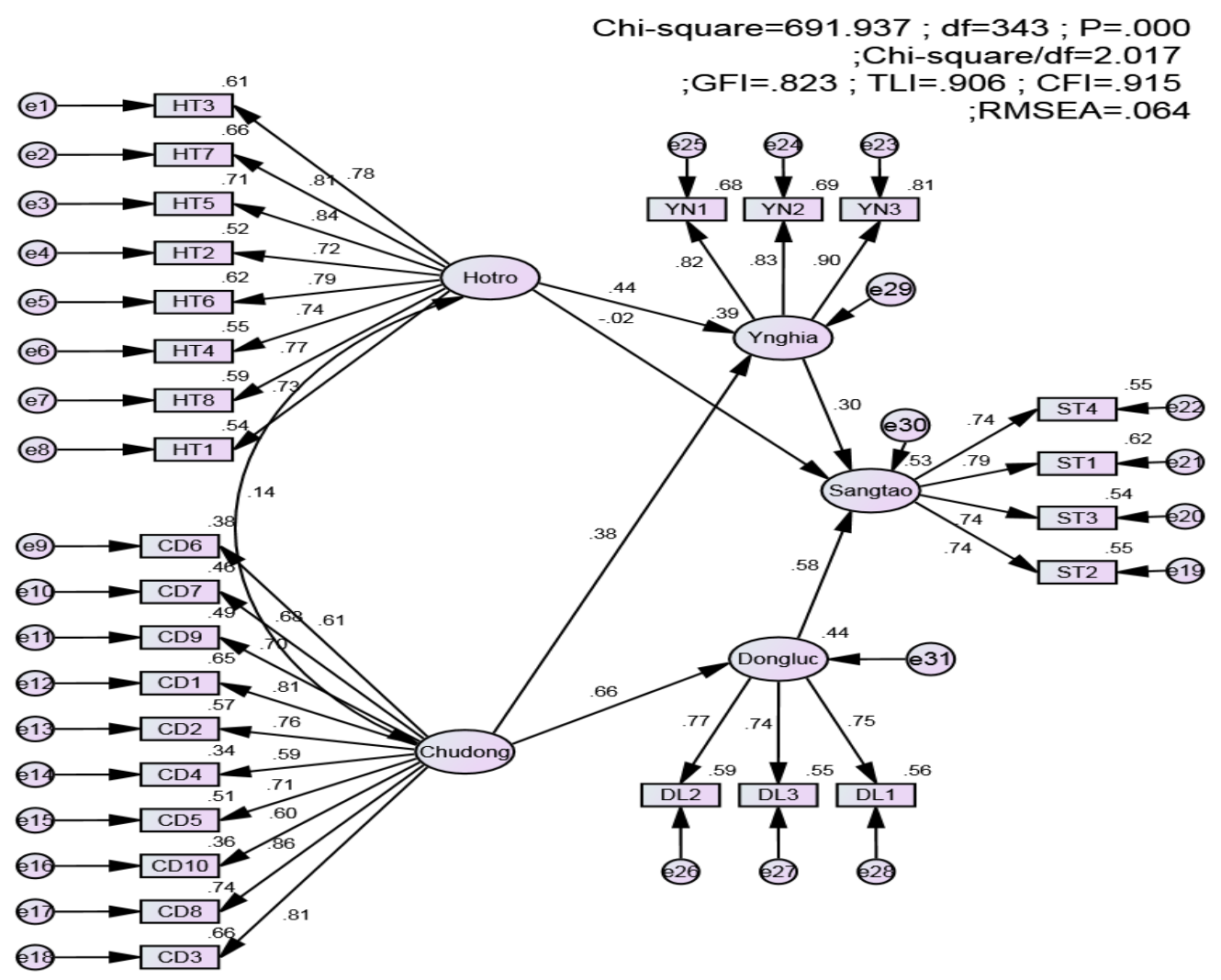

Hình 3. Mô hình phương trình cấu trúc (SEM) và hệ số ước lượng chuẩn hóa

\subsection{Kiểm định giả thuyết}

Bảng 5 thể hiện kết quả ước lượng mối quan hệ giữa các khái niệm nghiên cứu. Giả thuyết $\mathrm{H} 1$ cho rằng nhân tố sự hỗ trợ tổ chức có ảnh hưởng tích cực đến ý nghĩa công việc. Kết quả ước lượng SEM cho thấy nhân tố sự hỗ trợ tổ chức có mối quan hệ thuận chiều và tương quan mạnh với ý nghĩa công việc thể hiện qua hệ số ước lượng chuẩn hóa $\beta=0.445$; t-value $=$ $6.991>2.56$ và ước lượng này đạt ý nghĩa thống kê tại $p=0.000$. Điều này có nghĩa là người nhân viên sẽ nâng cao cảm nhận ý nghĩa công việc của mình nếu được sự quan tâm, ủng hộ của lãnh đạo đơn vị. Ngược lại, sự hỗ trợ tổ chức nghèo nàn, không đáp ứng nhu cầu dẫn đến mất ý nghĩa công việc của người nhân viên.

Kế tiếp, giả thuyết $\mathrm{H} 2$ cho rằng tính chủ động cá nhân có ảnh hưởng đến ý nghĩa công việc của người nhân viên. Kết quả phân tích cho thấy tính chủ động cá nhân có mối quan hệ thuận chiều và tích cực thông qua trọng số chuẩn hóa $\beta=0.383$; và giá trị t-value $=5.682>2.56$ và $\mathrm{p}=0.000<0.01$; đảm bảo ý nghĩa thống kê của mối quan hệ này với mức độ tin cậy $99 \%$. Điều này có nghĩa là tính chủ động cá nhân tăng thì ý nghĩa công việc gia tăng.

Giả thuyết $\mathrm{H} 3$ cho rằng yếu tố sự hỗ trợ tổ chức có ảnh hưởng đến sự sáng tạo của nhân viên. Thông qua kết quả phân tích cho thấy sự hỗ trợ tổ chức không ảnh hưởng đến sự sáng tạo của nhân viên, điều đó thể hiện qua giá trị t-value $=-0.229<1.96$ và $p=0.819>0.1$ nên không có ý nghĩa về mặt thống kê.

Giả thuyết 4 cho rằng nhân viên cảm thấy ý nghĩa công việc sẽ có ảnh hưởng đến sự sáng tạo đối với công việc. Điều này thể hiện qua hệ số tác động $\beta=0.305$ với giá trị t-value $=3.876$ $>1.96$ và giá trị $\mathrm{p}=0.000<0.01$. Do đó, giả thuyết $7 \mathrm{a}$ chứng minh rằng ý nghĩa công việc là yếu tố trung gian trong mối quan hệ giữa sự hỗ trợ của tổ chức và sự sáng tạo của nhân viên. Tác động gián tiếp của yếu tố ý nghĩa công việc có trọng số $\beta=0.104$ và đảm bảo ý nghĩa thống kê 
với mức độ tin cậy 99\% (p=0.001).

Giả thuyết $\mathrm{H} 5$ phát biểu rằng tính chủ động cá nhân có ảnh hưởng tích cực đến động lực nội tại của nhân viên. Mức độ ảnh hưởng là $\beta=0.662$. Giá trị $\mathrm{t}$-value $=7.492>2.56$ và ước lượng này đạt độ tin cậy $99 \%$. Điều này có nghĩa là khi nhân viên có tinh thần chủ động trong công việc thì động lực nội tại của người nhân viên sẽ được nâng cao.

Ngoài ra, giả thuyết 6 cho rằng động lực nội tại có ảnh hưởng đến sự sáng tạo của nhân viên, điều này thể hiện qua hệ số tác động $\beta=0.583$ và t-value $6.811>2.56$; ước lượng này có ý nghĩa thống kê $\mathrm{p}=0.000$. Chính vì vậy, giả thuyết được chấp nhận. Khi động lực nội tại được khuyến khích nhân viên dễ dàng sáng tạo, tiếp cận với những vấn đề mới, cập nhật tình hình thực hiện nghiên cứu của các chủ đề mà nhân viên quan tâm, từ đó sự sáng tạo sẽ được nâng cao.

Giả thuyết $7 \mathrm{~b}$ và $7 \mathrm{c}$ được chấp thuận và cho rằng ý nghĩa công việc và động lực nội tại là yếu tố trung gian trong mối quan hệ giữa tính chủ động cá nhân và sự sáng tạo của nhân viên. Mối quan hệ này đảm bảo ý nghĩa thống kê với mức độ tin cậy $99 \%(\mathrm{p}=0.001)$.

\section{Bảng 5}

Kết quả kiểm định các giả thuyết nghiên cứu

\begin{tabular}{|c|l|c|c|c|c|c|}
\hline $\begin{array}{c}\text { Giả } \\
\text { thuyết }\end{array}$ & \multicolumn{1}{|c|}{ Mối quan hệ } & $\begin{array}{c}\text { Hệ số ước } \\
\text { lượng } \\
\text { (beta) }\end{array}$ & $\begin{array}{c}\text { Hệ số ước } \\
\text { lượng } \\
\text { chuân hóa }\end{array}$ & $\begin{array}{c}\text { Sai số } \\
\text { chuâ̂n } \\
\text { (S.E) }\end{array}$ & $\begin{array}{c}\text { Kiểm } \\
\text { định t }\end{array}$ & $\begin{array}{c}\text { Mức ý } \\
\text { nghĩa } \\
\mathbf{p}\end{array}$ \\
\hline H1 & Hỗ trợ tổ chức $\rightarrow$ Ý nghĩa & 0.461 & 0.445 & 0.066 & 6.991 & $0.000^{* * *}$ \\
\hline H2 & Chủ động $\rightarrow$ Ý nghĩa & 0.580 & 0.383 & 0.102 & 5.682 & $0.000^{* * *}$ \\
\hline H3 & Hỗ trợ tổ chức $\rightarrow$ Sáng tạo & -0.012 & -0.016 & 0.051 & -0.229 & 0.819 \\
\hline H4 & Ý nghĩa $\rightarrow$ Sáng tạo & 0.220 & 0.305 & 0.057 & 3.876 & $0.000^{* * *}$ \\
\hline H5 & Chủ động $\rightarrow$ Động lực & 0.774 & 0.662 & 0.103 & 7.492 & $0.000^{* * *}$ \\
\hline H6 & Động lực $\rightarrow$ Sáng tạo & 0.546 & 0.583 & 0.080 & 6.811 & $0.000^{* * *}$ \\
\hline H7a & $\begin{array}{l}\text { Ảnh hưởng gián tiếp: } \\
\text { HT } \rightarrow \text { YN } \rightarrow \text { ST }\end{array}$ & 0,104 & 0,101 & 0.049 & 2.618 & $0.001 * * *$ \\
\hline H7b & $\begin{array}{l}\text { Ảnh hưởng gián tiếp: } \\
\text { CD } \rightarrow \text { YN } \rightarrow \text { ST }\end{array}$ & 0.127 & 0.120 & 0.051 & 2.597 & $0.001 * * *$ \\
\hline H7c & $\begin{array}{l}\text { Ảnh hưởng gián tiếp: } \\
\text { CD } \rightarrow \text { DL } \rightarrow \text { ST }\end{array}$ & 0.065 & 0.258 & 0.092 & 4.145 & $0.001 * * *$ \\
\hline
\end{tabular}

Ghi chú: *; **; *** lần lượt tương ứng với các mức ý nghĩa thống kê $10 \%, 5 \%$ và $1 \%$.

Nguồn: Kết quả nghiên cứu định tính và tính toán của nhóm tác giả

\section{Kết luận và hàm ý quản trị}

Kết quả nghiên cứu cho thấy bốn yếu tố tác động sự sáng tạo của nhân viên trong trường đại học công lập là động lực nội tại, tính chủ động cá nhân, sự hỗ trợ của tổ chức và ý nghĩa công việc.

Người nhân viên cần có động lực nội tại và cảm thấy ý nghĩa công việc mới góp phần nâng cao sự sáng tạo. Điều này chứng minh qua giả thuyết 4 và giả thuyết 6 . Tuy nhiên để nâng cao động lực nội tại và ý nghĩa công việc của người nhân viên cần có hai yếu tố: sự hỗ trợ của tổ chức (giả thuyết 1 ) và tính chủ động cá nhân (giả thuyết 2 , giả thuyết 5 ). Sự hỗ trợ tổ chức giúp 
người nhân viên cảm thấy nhận được ý nghĩa công việc và từ đó nâng cao sự sáng tạo (giả thuyết 7a). Người nhân viên cần rèn luyện tính chủ động trong công việc thì họ sẽ cảm thấy công việc có ý nghĩa (giả thuyết $7 \mathrm{~b}$ ) và thúc đẩy động lực nội tại (giả thuyết $7 \mathrm{c}$ ) điều này góp phần tăng thêm sự sáng tạo. Kết quả nghiên cứu cho thấy ý nghĩa công việc có vai trò trung gian mạnh hơn trong mối quan hệ giữa sự hỗ trợ của tổ chức đến sự sáng tạo của nhân viên, hơn là trong mối quan hệ giữa tính chủ động cá nhân và sáng tạo.

Kết quả bài nghiên cứu này cho thấy rằng trường đại học công lập tại Thành phố Hồ Chí Minh nên gia tăng sự hỗ trợ của tổ chức qua các hình thức khen thưởng, chia sẻ kiến thức giữa các nhà quản lý và nhân viên, và đồng thời khuyến khích gia tăng tính chủ động cá nhân. Các trường đại học nên xây dựng chương trình hành động để người nhân viên phát huy tính chủ động và động lực nội tại giúp gia tăng sự sáng tạo của nhân viên.

Tuy nhiên, phân tích thống kê cho thấy yếu tố sự hỗ trợ của tổ chức không tác động trực tiếp đến sự sáng tạo của nhân viên. Điều này khác biệt với hai nghiên cứu của Ibrahim và cộng sự (2016) và Horng và cộng sự (2016). Nghiên cứu của Ibrahim và cộng sự (2016) khảo sát nhân viên trong ngành sản xuất điện tử tại Malaysia và nghiên cứu của Horng và cộng sự (2016) thực hiện trong ngành du lịch tại Đài Loan. Sự khác biệt về đối tượng khảo sát và địa điểm khảo sát có thể dẫn tới kết quả nghiên cứu khác biệt. Thêm nữa, lý do sự khác biệt có thể từ mức độ của sự hỗ trợ của tổ chức trong các cơ sở đại học công lập tham gia khảo sát không hiện diện nhiều tại các tổ chức này. Nếu có sự hỗ trợ của tổ chức thì có thể do cơ chế quản lý nhà nước rườm rà nhiều thủ tục làm cho mức độ ảnh hưởng của sự hỗ trợ tổ chức không phát huy đáng kể. Tuy nhiên, sự hỗ trợ của tổ chức vẫn là một yếu tố rất quan trọng, và ảnh hưởng gián tiếp đến sự sáng tạo vì giúp người nhân viên cảm thấy có ý nghĩa công việc.

Các trường đại học công lập hiện nay thể hiện những đặc điểm tương tự mẫu nghiên cứu, đa số là nhân viên, trẻ tuổi, mức thu nhập trung bình. Chính sách, giải pháp nâng cao sự sáng tạo của người nhân viên tại các trường đại học có thể hướng đến các nội dung sau:

Thứ nhất giải pháp gia tăng tính chủ động cá nhân (yếu tố quan trọng giúp cải thiện ý nghĩa công việc và động lực nội tại): Các trường đại học công lập cần tạo ra các chương trình hành động khuyến khích nhân viên chấp nhận thử thách, làm cho nhân viên cảm nhận được vai trò quan trọng của mình trong tổ chức để luôn chủ động, mạnh dạn đưa ra ý kiến, tổ chức hội thảo cho nhân viên tham gia xây dựng chiến lược cũng như những quyết định quan trọng của tổ chức bao gồm phổ biến lộ trình thăng tiến, chính sách đào tạo - bồi dưỡng, chính sách khen thưởng - kỷ luật, quy trình đánh giá viên chức rõ ràng.

Thứ hai giải pháp gia tăng động lực nội tại, các trường đại học cần tổ chức khuyến khích, động viên, thấu hiểu nhân viên, phân công công việc đúng và rõ ràng.

Thứ ba gia tăng sự hỗ trợ của tổ chức: Trường đại học cần tạo môi trường làm việc thân thiện khuyến khích sáng tạo, đổi mới, để nhân viên dám đổi mới trong quy trình làm việc, trong các đề xuất với lãnh đạo; thể hiện cho nhân viên thấy sự quan tâm của lãnh đạo, sự ghi nhận thành quả và được tôn trọng; thay đổi công việc giúp cho nhân viên không rơi vào giai đoạn nhàm chán.

Bài nghiên cứu này thực hiện đầy đủ các bước, và có cơ sở lý thuyết đáng tin cậy và đối tượng khảo sát đúng yêu cầu. Hạn chế của bài viết là mẫu khảo sát là những người giữ chức vụ quản lý còn khá khiêm tốn. Hạn chế thứ hai là về cách thức khảo sát không áp dụng khảo sát trực tiếp từng cá nhân để đánh giá mức độ trung thực của người trả lời và mức độ hiểu câu hỏi của họ. Định hướng nghiên cứu sau này có thể bổ sung các yếu tố khác như môi trường làm việc, tài chính, cơ chế lương thưởng ảnh hưởng thế nào đến sự sáng tạo của nhân viên nhằm xây dựng giải pháp tăng cường năng lực sáng tạo càng đầy đủ và phù hợp. 


\section{Tài liệu tham khảo}

Akgunduz, Y., Alkan, C., \& Gök, Ö. A. (2018). Perceived organizational support, employee creativity and proactive personality: The mediating effect of meaning of work. Journal of Hospitality and Tourism Management, 34(1), 105-114. doi:10.1016/j.jhtm.2018.01.004

Amabile, T. M. (1983). The social psychology of creativity: A componential conceptualization. Journal of Personality and Social Psychology, 45(2), 357-376. doi:10.1037/00223514.45.2.357

Amabile, T. M. (1988). A model of creativity and innovation in organizations. Research in Organizational Behavior, 10(1), 123-167.

Amabile, T. M. (1997). Motivating creativity in organizations: On doing what you love and loving what you do. California Management Review, 40(1), 39-58. doi:10.2307/41165921

Amabile, T. M. (2012). Componential theory of creativity. In E. H. Kessler (Ed.), Encyclopedia of management theory (pp. 1-10). Boston, MA: Harvard Business School Working Paper.

Amabile, T. M., Barsade, S. G., Mueller, J. S., \& Staw, B. M. (2005). Affect and creativity at work. Administrative Science Quarterly, 50(3), 367-403. doi:10.2189/asqu.2005.50.3.367

Amabile, T. M., Conti, R., Coon, H., Lazenby, J., \& Herron, M. (1996). Assessing the work environment for creativity. Academy of Management Journal, 39(5), 1154-1184. doi: $10.5465 / 256995$

Anderson, J. C., \& Gerbing, D. W. (1988). Structural equation modeling in practice: A review and recommended two-step approach. Psychological Bulletin, 103(3), 411-423. doi:10.1037//0033-2909.103.3.411

Asyraf, W. M., \& Afthanorhan, B. Q. (2013). A comparison of Partial Least Square Structural Equation Modeling (PLS-SEM) and covariance based structural equation modeling (CBSEM) for confirmatory factor analysis. International Journal of Engineering Science and Innovative Technology, 2(5), 198-205.

Bảo Hiểm Xã Hội. (2015). Chất lương nguồn nhân lực Việt Nam chi đạt 3.79/10 điểm [The quality of human resources in Vietnam is only 3.79/10 points]. Retrieved April 25, 2019, from http://tapchibaohiemxahoi.gov.vn/tin-tuc/chat-luong-nguon-nhan-luc-viet-nam-chidat-37910-diem-8849

Bateman, T. S., \& Crant, J. M. (1993). The proactive component of organizational behavior: A measure and correlates. Journal of Organizational Behavior, 14(2), 103-118. doi:10.1002/job.4030140202

Baumgartner, H., \& Homburg, C. (1996). Applications of structural equation modeling in marketing and consumer research: A review. International Journal of Research in Marketing, 13(2), 139-161. doi:10.1016/0167-8116(95)00038-0

Blau, P. (1964). Exchange and power in social life. New York, NY: Wiley.

Bui, H. Q., \& Nguyen, B. T. (2019). Các yếu tố ảnh hưởng đến động lực làm việc của công chức tại cuc thué Thành phố Hồ Chí Minh [Factors affecting the working motivation of civil servants at Ho Chi Minh City tax department]. Retrieved April 25, 2019, from http://tapchitaichinh.vn/nghien-cuu-trao-doi/cac-yeu-to-anh-huong-den-dong-luc-lam-vieccua-cong-chuc-tai-cuc-thue-tp-ho-chi-minh-302420.html

Coelho, F., Augusto, M., \& Lages, L. F. (2011). Contextual factors and the creativity of frontline employees: The mediating effects of role stress and intrinsic motivation. Journal of Tetailing, 87(1), 31-45. doi:10.1016/j.jretai.2010.11.004 
Dawson, P., \& Andriopoulos, C. (2014). Managing change, creativity and innovation. London, UK: Sage Publications Ltd.

DiLiello, T. C., \& Houghton, J. D. (2006). Maximizing organizational leadership capacity for the future: Toward a model of self-leadership, innovation and creativity. Journal of Managerial Psychology, 21(4), 319-337. doi:10.1108/02683940610663114

Dul, J., \& Ceylan, C. (2011). Work environments for employee creativity. Ergonomics, 54(1), 12-20. doi:10.1080/00140139.2010.542833

Eder, P., \& Sawyer, J. (2008). The power to be creative at work: examining the componential model of employee creativity. Eastern Academy of Management Annual Conference in Washington, DC, 71(3), 500-507.

Eisenberger, R., Huntington, R., Hutchison, S., \& Sowa, D. (1986). Perceived organizational support. Journal of Applied Psychology, 71(3), 500-507. doi:10.1037/0021-9010.71.3.500

Ha, G. N. K., \& Nguyen, V. H. (2015). Các yếu tố ảnh hưởng đến sự sáng tạo của nhân viên trong công việc tại trường Đại học Tài chính - Marketing [Factors affecting the creativity of employees at work at University of Finance - Marketing]. Nghiên cúu Tài chính Marketing, 31(12), 22-34.

Hair, J. F., Black, W. C., Babin, B. J., \& Anderson, R. E. (2010). Multivariate data analysis. Upper Saddle River, NJ: Prentice Hall.

Hair, J. F., Black, W. C., Babin, B. J., Anderson, R. E., \& Tatham, R. L. (2006). Multivariate data analysis. Upper Saddle River, NJ: Person Prentice Hall.

Hair, J. F., Gabriel, M. L. D. S., \& Patel, V. K. (2014). AMOS CB-SEM: Guidelines on its application as marketing research tool. Brazilian Journal of Marketing - BJM. 13, 44-55 doi:10.5585/remark.v13i2.2718

Horng, J. S., Tsai, C. Y., Yang, T. C., Liu, C. H., \& Hu, D. C. (2016). Exploring the relationship between proactive personality, work environment and employee creativity among tourism and hospitality employees. International Journal of Hospitality Management, 54(2016), 25-34. doi:10.1016/j.ijhm.2016.01.004

Houghton, J. D., \& DiLiello, T. C. (2010). Leadership development: The key to unlocking individual creativity in organizations. Leadership and Organization Development Journal, 31(3), 230-245. doi:10.1108/01437731011039343

Hulland, J. (1999). Use of Partial Least Squares (PLS) in strategic management research: A review of four recent studies. Strategic Management Journal, 20(2), 195-204. doi:10.1002/(SICI)1097-0266(199902)20:2<195::AID-SMJ13>3.0.CO;2-7

Ibrahim, H. I., Isa, A., \& Shahbudin, A. S. M. (2016). Organizational support and creativity: The role of developmental experiences as a moderator. Procedia Economics and Finance, 35, 509-514. doi:10.1016/s2212-5671(16)00063-0

Jaiswal, N. K., \& Dhar, R. L. (2015). Transformational leadership, innovation climate, creative self-efficacy and employee creativity: A multilevel study. International Journal of Hospitality Management, 51, 30-41. doi:10.1016/j.ijhm.2015.07.002

Joo, B. K. B., Hahn, H. J., \& Petersonb, S. L. (2015). Turnover intention: The effects of core self-evaluations, proactive personality, perceived organizational support, developmental feedback, and job complexity. Human Resource Development International, 18(2), 116130. doi:10.1080/13678868.2015.1026549 
Joo, B. K. B., \& Lim, T. (2009). The effects of organizational learning culture, perceived job complexity, and proactive personality on organizational commitment and intrinsic motivation. Journal of Leadership and Organizational Studies, 16(1), 48-60. doi:10.1177/1548051809334195

Kline, R. B. (2011). Principles and practice of structural equation modeling (3rd ed.). New York, NY: Guilford Press.

Luecke, R., \& Katz, R. (2004). Managing creativity and innovation. Boston, MA: Harvard Business School Press.

MacCallum, R. C., Browne, M. W., \& Sugawara, H. M. (1996). Power analysis and determination of sample size for covariance structure modeling. Psychological Methods, 1(2), 130-149. doi:10.1037/1082-989X.1.2.130

Nguyen, N. D., \& Le, L. P. (2017). Các yếu tố tính cách ảnh hưởng đến năng lực sáng tạo của nhân viên tại các doanh nghiệp ở Thành phố Hồ Chí Minh [Personality factors affecting the creative ability of employees at enterprises in Ho Chi Minh City]. Tạp chi Khoa hoc Đại học Mở Thành phố Hồ Chí Minh, 54(3), 102-119.

Nguyen, T. D. (2011). Phương pháp nghiên cưu khoa học trong kinh doanh [Scientific research methods in business]. Hanoi, Vietnam: Nhà xuất bản Lao Động- Xã hội.

Nguyen, T. D. (2014). Giáo trình phuoong pháp nghiên cúu khoa học trong kinh doanh [Textbook of scientific research methods in business]. Hanoi, Vietnam: Nhà Xuất Bản Tài Chính.

Shalley, C. E., Zhou, J., \& Oldham, G. R. (2004). The effects of personal and contextual characteristics on creativity: Where should we go from here? Journal of Management, 30(6), 933-958. doi:10.1016/j.jm.2004.06.007

Spreitzer, G. M. (1995). Psychological empowerment in the workplace: Dimensions, measurement, and validation. Academy of Management Journal, 38(5), 1442-1465. doi: $10.5465 / 256865$

Tran, D. V. (2013). Một số suy nghĩ về năng lực sáng tạo và phương hướng năng lực sáng tạo của con người Việt Nam [Some thoughts on creative capacity and direction of creative capacity of Vietnamese people]. Tạp chí Khoa học trưòng Đại học Su phạm, 49.

Woodman, R. W., Sawyer, J. E., \& Griffin, R. W. (1993). Toward a theory of organizational creativity. Academy of Management Review, 18(2), 293-321. doi:10.5465/amr.1993.3997517

Zhang, X., \& Bartol, K. M. (2010). Linking empowering leadership and employee creativity: The influence of psychological empowerment, intrinsic motivation, and creative process engagement. Academy of Management Journal, 53(1), 107-128. doi:10.5465/amj.2010.48037118

Zhou, J., \& George, J. M. (2001). When job dissatisfaction leads to creativity: Encouraging the expression of voice. Academy of Management Journal, 44(4), 682-696. doi: $10.2307 / 306941$

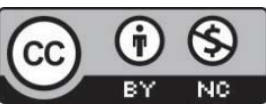

